\title{
Caracterização hidrodinâmica e térmica de um solo de Mata Atlântica
}

\author{
Hydrodynamic and thermal characterization of an Atlantic Forest \\ Caracterización hidrodinámica y térmica de un suelo de Mata Atlántica
}

Recebido: 23/04/2021 | Revisado: 29/04/2021 | Aceito: 03/05/2021 | Publicado: 16/05/2021

\author{
Ariela Rocha Cavalcanti \\ ORCID: https://orcid.org/0000-0003-2252-1459 \\ Universidade de Pernambuco, Brasil \\ E-mail: arc_pec@poli.br \\ Willames de Albuquerque Soares \\ ORCID: https://orcid.org/0000-0003-3268-7241 \\ Universidade de Pernambuco, Brasil \\ E-mail: was@poli.br \\ Marco Aurélio Calixto Ribeiro de Holanda \\ ORCID: https://orcid.org/0000-0002-0860-2458 \\ Universidade Federal de Pernambuco, Brasil \\ E-mail: holandamacr@yahoo.com.br
}

\begin{abstract}
Resumo
As Caracterizações hidrodinâmica e térmica do solo são indispensáveis para subsidiar pesquisas relacionadas a análise dos processos de interação solo-planta-atmosfera. Deste modo, o intuito desta pesquisa é realizar a caracterização hidrodinâmica e térmica de um solo que compõe um fragmento de Mata Atlântica, localizado no bairro de Dois Irmãos, Recife, Pernambuco, Brasil. Para tanto, foram realizados ensaios laboratoriais de granulometria e ensaios de infiltração utilizando-se uma coluna de solo. Para obtenção dos parâmetros hidrodinâmicos seguiu-se a metodologia Beerkan Estimation of Soil Transfer (BEST), enquanto que as propriedades térmicas do solo foram obtidas a partir de modelos propostos por De Vries (1963). Após estes procedimentos, o solo em análise foi classificado como franco arenoso. Verificou-se que o BEST forneceu valores coerentes para os parâmetros de forma e de normalização das curvas de retenção da água no solo e de condutividade hidráulica. Com relação às propriedades térmicas do solo observou-se uma significativa variação com o aumento da umidade, ficando evidente que a água no solo desempenha um papel determinante na dinâmica da propagação do calor. Os valores das propriedades hidrodinâmicas e térmicas do solo, fornecidos pelos diferentes métodos empregados, encontraram-se dentro da faixa estabelecida na literatura.
\end{abstract}

Palavras-chave: BEST; Infiltração; Modelo de De Vries; Propagação de calor.

\begin{abstract}
Soil hydrodynamic and thermal characterization are indispensable to support research related to the analysis of soilplant-atmosphere interaction processes. Therefore, the objective was to carry out the hydrodynamic and thermal characterization of a soil that makes up a fragment of the Atlantic Forest, located in the Dois Irmãos neighborhood, in Recife, Pernambuco, Brazil. For this purpose, laboratory tests of granulometry and infiltration tests using a soil column. To obtain the hydrodynamic parameters, the Beerkan Estimation of Soil Transfer (BEST) methodology was followed, while the thermal properties of the soil were obtained from models proposed by De Vries (1963). After laboratory tests, the soil under analysis was classified as sandy loam. It was found that BEST provided coherent values for the shape and normalization parameters of the soil water retention and hydraulic conductivity curves. Regarding the thermal properties of the soil, a significant variation was observed with the increase in humidity, making it evident that water in the soil plays a determining role in the dynamics of heat propagation. The values of the hydrodynamic and thermal properties of the soil, provided by the different methods employed, were within the range established in the literature
\end{abstract}

Keywords: BEST; Infiltration; De Vries model; Heat propagation.

\section{Resumen}

Las caracterizaciones hidrodinámicas y térmicas del suelo son indispensables para apoyar la investigación relacionada con el análisis de los procesos de interacción suelo-planta-atmósfera. Así, el objetivo fue realizar la caracterización hidrodinámica y térmica de un suelo que compone un fragmento de la Mata Atlántica, ubicado en el barrio Dois Irmãos, Recife, Pernambuco, Brasil. Para ello, se realizaron pruebas de laboratorio de granulometría y pruebas de infiltración mediante columna de suelo. Para la obtención de los parámetros hidrodinámicos se siguió la metodología Beerkan Estimation of Soil Transfer (BEST), mientras que las propiedades térmicas del suelo se obtuvieron a partir de modelos propuestos por De Vries (1963). Después de las pruebas de laboratorio, el suelo analizado se clasificó como franco arenoso. Se encontró que BEST proporcionó valores coherentes para la forma y los parámetros de normalización de las curvas de retención de agua del suelo y conductividad hidráulica. En cuanto a las propiedades 
térmicas del suelo, se observó una variación significativa con el aumento de la humedad, evidenciando que el agua en el suelo juega un papel determinante en la dinámica de propagación del calor. Los valores de las propiedades hidrodinámicas y térmicas del suelo, proporcionados por los diferentes métodos empleados, estuvieron dentro del rango establecido en la literatura.

Palabras clave: BEST; Infiltración; Modelo De Vries; Propagación de calor.

\section{Introdução}

A Mata Atlântica é uma floresta tropical que originalmente cobria cerca de $1.345 .300 \mathrm{~km}^{2}$, distribuídos em três países (92\% da área total no Brasil e os $8 \%$ restantes no Paraguai e Argentina). Atualmente, seus remanescentes florestais representam 12,4\% da área original, cobrindo aproximadamente 17\% do território brasileiro, de Nordeste a Sudeste (Santana et al., 2020). Por muitos anos foi intensamente desmatada com os ciclos econômicos e o processo de urbanização. Estima-se que a floresta foi reduzida a 250.000 fragmentos, destes apenas 42.500 apresentam área superior a 50 ha. Estes fragmentos de Mata Atlântica urbanos proporcionam mais equilíbrio nas condições climáticas locais, amenizando os efeitos causados nas cidades por precipitações e temperaturas elevadas (Lima et al., 2018).

$\mathrm{Na}$ análise dos processos de interação solo-planta-atmosfera, o conhecimento dos regimes hídrico e térmico de um local assume grande importância, fazendo-se necessário conhecer as propriedades hidrodinâmicas e térmicas do solo (Kelleners et al., 2016). Essas propriedades servem como subsídio para pesquisas relacionadas a modelagem do sistema soloplanta-atmosfera, estudo da dinâmica da água, do calor e solutos em meios porosos, a partição da chuva na infiltração e escoamento superficial, além de auxiliar na análise de mudanças climáticas e os impactos gerados pela ação antrópica ao meio ambiente (Castellini et al., 2021).

As curvas de retenção da água e de condutividade hidráulica do solo representam, respectivamente, sua capacidade de armazenamento e a facilidade com que a água é conduzida através dele (Hillel, 1998). Já as propriedades térmicas que governam a transferência de calor no solo são: a condutividade térmica aparente, a capacidade térmica volumétrica e a difusividade térmica aparente, as quais são resultantes de um conjunto de fatores, incluindo sua textura, estrutura, temperatura e o teor volumétrico de água (Zhao et al., 2019).

Determinar as propriedades hidrodinâmicas e térmicas do solo pode requerer ensaios de campo e de laboratório bastante dispendiosos, onerando e demandando um longo tempo de execução. Por esses motivos e pela importância da análise da dinâmica da água e do calor no solo, alguns pesquisadores desenvolveram métodos mais simples e que requerem dados do solo mais fáceis de serem obtidos, mais usuais e de baixo custo (Ewing e Horton, 2007).

Segundo Lassabatère et al. (2006), vários métodos têm sido desenvolvidos para determinar as propriedades hidrodinâmicas do solo. Os mais simples requerem informações da distribuição de tamanho de partículas e de medições de campo simples. De forma semelhante, as propriedades térmicas do solo podem ser determinadas através de diversos modelos presentes na literatura, os quais, em sua maioria, exigem o conhecimento das propriedades físicas e das frações volumétricas dos componentes do solo. A exemplo, tem-se os modelos propostos por De Vries (1963), utilizado nas obtenções da condutividade térmica aparente e da capacidade térmica volumétrica do solo, os quais já foram amplamente utilizados com considerável precisão (Zhao et al., 2019).

Sabendo-se da sua importância e que cada tipo de solo possui características específicas, faz-se necessário que sejam identificadas para cada situação particular as suas propriedades hidrodinâmicas e térmicas. Embora haja alguns estudos sobre processos hidrológicos e análise de variabilidade de temperatura em solos conduzidos em áreas de Mata Atlântica (Freitas et al., 2013; Carneiro et al., 2014), pouco se conhece sobre a dinâmica do transporte de água e calor que ocorre nestes solos. Deste modo, o objetivo desta pesquisa é realizar a caracterização hidrodinâmica e térmica de um solo que compõe um fragmento de Mata Atlântica, a fim de facilitar o entendimento e subsidiar estudos que venham a contribuir para o adequado 
manejo e preservação de áreas sob essas condições ambientais.

\section{Metodologia}

\subsection{Descrição da área de Coleta}

Os dados utilizados no estudo foram obtidos a partir de uma amostra de solo coletada em um fragmento de Mata Atlântica de aproximadamente 384,42 ha, localizado no bairro de Dois Irmãos, Recife, Pernambuco, Brasil. O local possui as seguintes coordenadas geográficas: ao Sul, Latitude - 08 $1^{\prime} 12.675^{\prime \prime}$ e ao Oeste, Longitude - 34 $57^{\prime} 8.80704 "$ ", com altitude de 13,24 m em relação ao nível do mar (Figura 1). Segundo a classificação climática de Köppen, a cidade do Recife tem um clima As' caracterizado como tropical atlântico de costa oriental, com chuvas de outono-inverno.

Figura 1. Localização espacial da área de coleta.

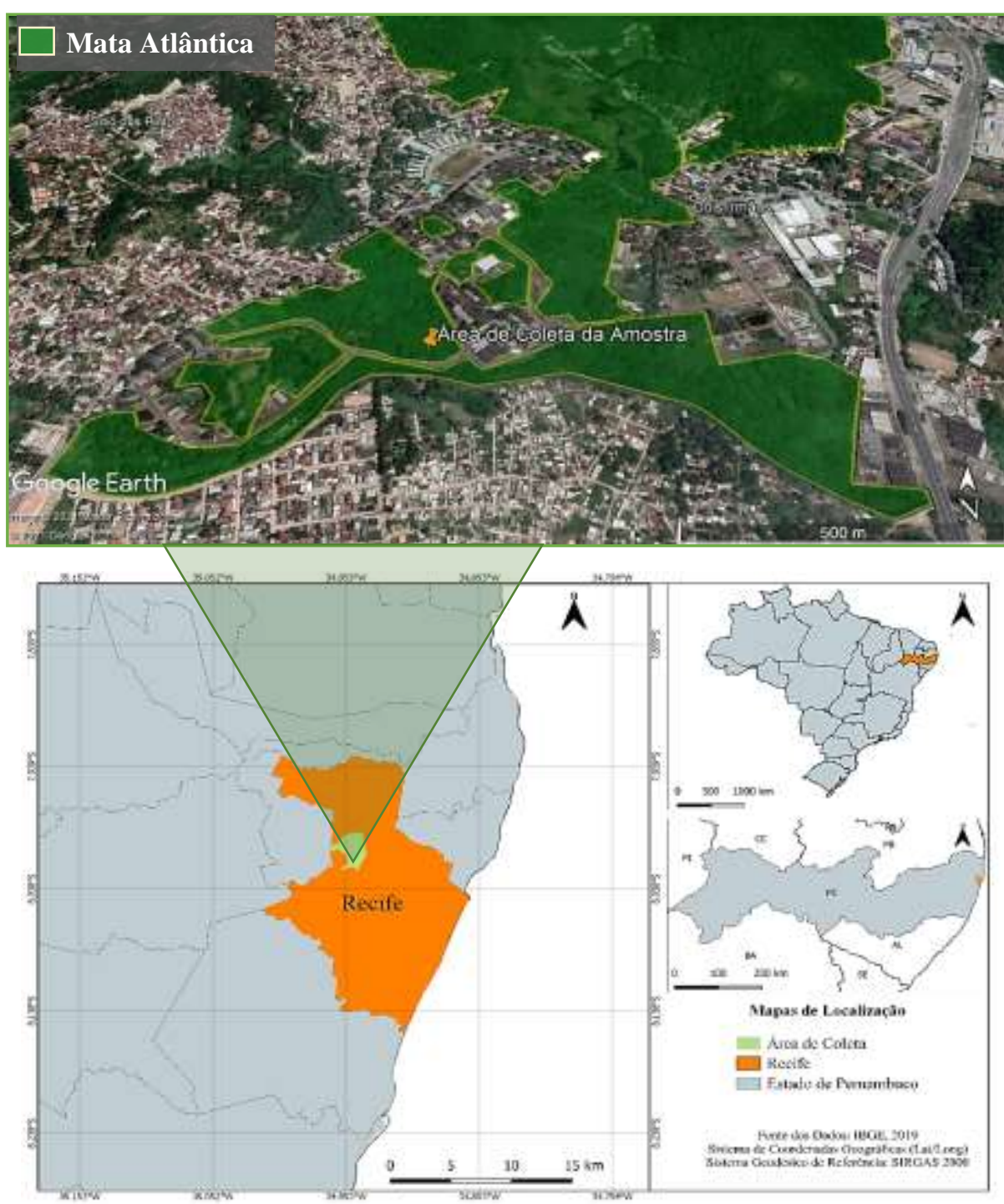

Fonte: Autores.

\subsection{Ensaios Laboratoriais}

Foram realizados ensaios de caracterização física do solo mediante análise granulométrica realizada de acordo com as normas estabelecidas pela NBR-7181 (ABNT, 2016), a qual permite determinar os diâmetros das partículas mais finas (argila e silte) por sedimentação e as mais grosseiras (areia) por peneiramento. Para a determinação do teor de matéria orgânica $\left(M_{o}\right)$ 
seguiu-se as recomendações da NBR-13600 (ABNT, 1996) e a densidade das partículas do solo $\left(\rho_{p}\right)$ foi obtida segundo Teixeira et al. (2017).

Para a obtenção de parâmetros hidrodinâmicos do solo, foram realizados ensaios laboratoriais de infiltração utilizando-se volumes idênticos de água $(15 \mathrm{ml})$, um infiltrômetro de anel simples de $70 \mathrm{~mm}$ de diâmetro e uma coluna de solo, segundo o método Beerkan (Haverkamp et al., 1994). Esta coluna foi montada em um tubo de acrílico de $188 \mathrm{~mm}$ de diâmetro interno e $350 \mathrm{~mm}$ de altura, dos quais $40 \mathrm{~mm}$ são referentes a camada de drenagem, $270 \mathrm{~mm}$ de solo e o espaço restante (40 $\mathrm{mm}$ ) foi utilizado para alocação do infiltrômetro (Figura 2). Para garantir que a coluna mantivesse sua densidade aparente uniforme, o solo foi sendo introduzido aos poucos ( $140 \mathrm{~g}$ por vez) e entre uma adição e outra era realizada uma compactação.

Figura 2. Desenho esquemático da coluna de solo e aparato experimental (dimensões em milímetros).
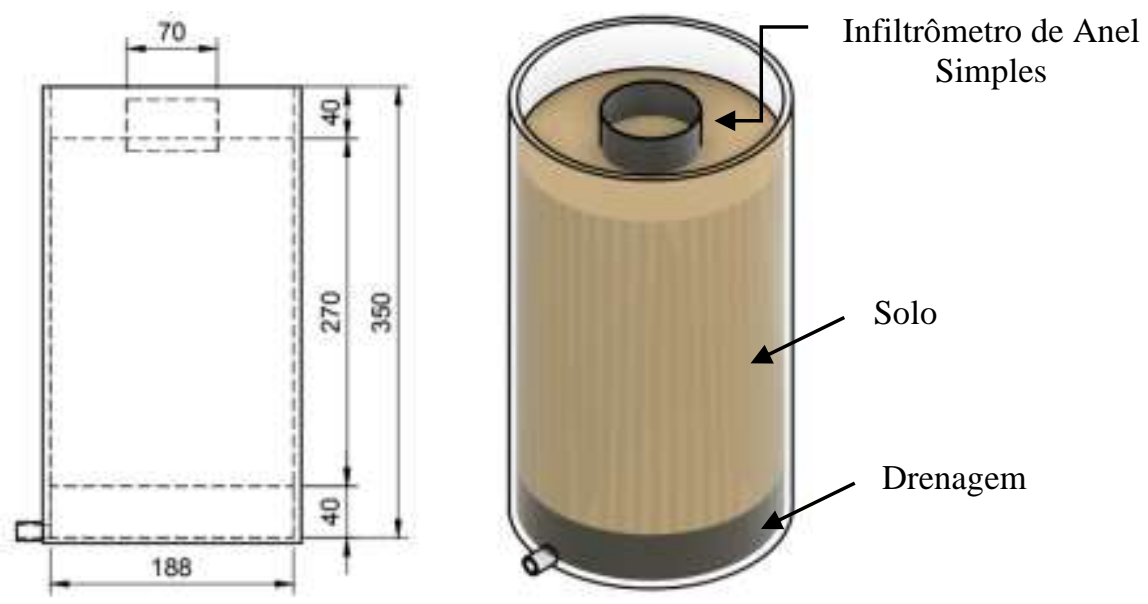

Fonte: Autores.

Durante a montagem da coluna, foi separada uma amostra de $140 \mathrm{~g}$ de solo, exposta às mesmas condições ambientais da coluna, até o momento em que o ensaio de infiltração se iniciou, quando esta amostra foi selada para posterior obtenção da umidade volumétrica inicial $\left(\theta_{0}\right)$. Ao final do procedimento de infiltração foi coletada outra amostra de solo de dentro da coluna para determinação da umidade volumétrica final $\left(\theta_{f}\right)$. Além disso, a título de triplicata, foram realizadas três repetições do ensaio de infiltração e utilizada a média dos valores obtidos, sendo a coluna de solo desmontada e remontada para cada repetição. A não realização de ensaios de infiltração in situ deu-se pela elevada umidade do solo da Mata Atlântica, o que viria a desrespeitar a restrição imposta por Haverkamp et al. (1994), a qual diz que a razão entre $\theta_{0}$ e $\theta_{f}$ deve ser inferior a $25 \%$ para um melhor ajuste dos dados experimentais.

\subsection{Caracterização Hidrodinâmica do Solo}

Os ensaios granulométricos e de infiltração originam os dados de entrada para a estimativa dos parâmetros de forma $(n, m$ e $\eta)$ e de normalização $\left(\theta_{s}, k_{s}\right.$ e $\left.h_{g}\right)$ das curvas de retenção da água $\theta(h)$ e de condutividade hidráulica no solo $K(\theta)$. Na obtenção desses parâmetros foi utilizado um método específico baseado na metodologia Beerkan, intitulado Beerkan Estimation of Soil Transfer (BEST), proposto por Lassabatère et al. (2006). Neste método, $\theta(h)$ e $K(\theta)$ são descritas pelos modelos de van Genuchten (1980) e de Brooks e Corey (1964), Equações (1) e (2), respectivamente. 


$$
\begin{gathered}
\theta(h)=\theta_{r}+\frac{\theta_{s}-\theta_{r}}{\left(1+\left|\frac{h}{h_{g}}\right|^{n}\right)^{m}}, \operatorname{com} m=1-\frac{2}{n} \\
K(\theta)=k_{s}\left(\frac{\theta-\theta_{r}}{\theta_{s}-\theta_{r}}\right)^{\eta}, \operatorname{com} \eta=\frac{2}{n m}+3
\end{gathered}
$$

em que: $\theta$ é a umidade volumétrica $\left(\mathrm{mm}^{3} \mathrm{~mm}^{-3}\right) ; \theta_{r}$ e $\theta_{s}$ as umidades volumétricas residual e saturada, respectivamente $\left(\mathrm{mm}^{3}\right.$ $\left.\mathrm{mm}^{-3}\right) ; h$ o potencial matricial $(\mathrm{mm}) ; h_{g}$ um valor de escala de $h(\mathrm{~mm}) ; k_{s}$ a condutividade hidráulica saturada $\left(\mathrm{mm} \mathrm{s}^{-1}\right) ;$ e $n, m$ e $\eta$ os parâmetros de forma, mediante hipótese de Burdine (1953). Por meio do BEST, estes últimos parâmetros são estimados a partir da distribuição granulométrica do solo $(F(D))$ e a sua porosidade total $(\varepsilon)$, obtida pela razão entre a densidade aparente do solo $\left(\rho_{b}\right)$ e das partículas $\left(\rho_{p}\right)$. No emprego do BEST considera-se $\theta_{r}$ igual a zero e $\theta_{s}$ foi considerado de valor igual a $\varepsilon$. Os parâmetros de normalização $k_{s}$ e $h_{g}$ foram obtidos a partir dos experimentos de infiltração tridimensional. A metodologia detalhada para a determinação dos parâmetros de forma e de normalização encontra-se em Lassabatère et al. (2006).

\subsection{Caracterização Térmica do Solo}

A estimativa das propriedades térmicas do solo foi realizada mediante os dados obtidos após sua caracterização física. Foram considerados os modelos propostos por De Vries (1963) para obtenção da condutividade térmica aparente $(\lambda)$ e da capacidade térmica volumétrica do solo $(\mu)$. A difusividade térmica aparente $(\alpha)$ é o parâmetro que relaciona a capacidade de condução (condutividade térmica aparente) ao armazenamento de calor (capacidade térmica volumétrica), podendo, por definição, ser obtida pela razão entre $\lambda$ e $\mu$.

Neste caso, para obtenção de $\lambda$, De Vries (1963) considerou o solo como uma mistura de partículas elipsoidais rodeadas por um meio contínuo de ar ou água. A condutividade térmica aparente do meio é obtida como uma combinação de todos os componentes que o constituem: minerais, matéria orgânica, ar e água. A estimativa de $\lambda$ é realizada conforme a Equação (3).

$$
\lambda=\frac{\sum_{i} k_{i} \lambda_{i} \theta_{i}}{\sum_{i} k_{i} \theta_{i}}
$$

em que: $\theta_{i}$ é a fração volumétrica de cada constituinte $i(\%)$, considerando-se, no caso dos minerais, os teores de areia, silte e argila. Já para o ar, foi considerado o volume dos poros do solo não preenchidos por água; $\lambda_{i}$ é a condutividade térmica de cada constituinte $i\left(\mathrm{~W} \mathrm{~m}^{-1} \mathrm{~K}^{-1}\right)$, que tem seus valores tabelados; e $k_{i}$ é o fator de ponderação que depende da forma e da orientação dos grânulos dos constituintes do solo e da relação das condutividades dos constituintes, como mostra a Equação (4).

$$
k_{i}=\frac{1}{3} \sum_{i=a, b, c}\left[1+\left(\frac{\lambda_{i}}{\lambda_{o}}-1\right) g_{i}\right]^{-1}
$$

$\lambda_{o}$ é a condutividade térmica da fase contínua (água ou ar, para quando o solo está seco). Os valores de $g_{i}$, que foram originalmente planejados para serem fatores de forma das partículas elipsoidais, são utilizados como parâmetros para ajustar os 
dados empíricos, onde $g_{a}+g_{b}+g_{c}=1$, sendo $a, b, c$ a representação dos eixos da elipsoide. No emprego deste modelo, presume-se que $g_{a}$ e $g_{b}$ são iguais, de modo que $g_{c}=1-2 g_{a}$, fazendo com que apenas um fator de forma precise ser calculado para cada constituinte. O parâmetro $g_{a}$ foi obtido conforme Tian et al. (2016) e a temperatura média foi determinada por meio de um sensor inserido na coluna de solo (Figura 2) a uma profundidade de 0,14 m, com medições realizadas a cada 10 segundos durante 15 dias. Para os fins deste estudo, assumiu-se que a fase contínua era a água e sua fração volumétrica variou da umidade critica a umidade saturada. Segundo De Vries (1963), o valor da umidade crítica é de aproximadamente $0,03 \mathrm{~m}^{3} \mathrm{~m}^{-3}$ para solos de textura grossa e entre 0,05 e $0,10 \mathrm{~m}^{3} \mathrm{~m}^{-3}$ para solos de textura fina.

Para obtenção de capacidade térmica volumétrica do solo $(\mu)$, foi utilizada uma abordagem comumente creditada a De Vries (1963). Neste método, pondera-se os diferentes constituintes do solo por suas frações volumétricas e adota-se os valores numéricos das capacidades caloríficas desses componentes, conforme Equação (5).

$$
\mu=1,92 f_{m}+2,51 M_{o}+4,18 \theta
$$

em que: $f_{m}$ é a fração de volume dos minerais, obtida a partir da Equação (6).

$$
f_{m}=1-\left(\varepsilon+M_{o}\right)
$$

\section{Resultados}

Mediante a caracterização física do solo, foi possível a obtenção dos percentuais médios de areia, silte e argila, além dos valores de $M_{o}, f_{m}, \varepsilon, \rho_{b}, \rho_{p}$ e respectiva classificação textural, conforme Tabela 1. Também são apresentados os valores referentes a $\theta_{0}, \theta_{f}$, ao tempo total de infiltração, a infiltração cumulativa e a média da taxa de infiltração, obtidos após ensaio de infiltração.

\begin{tabular}{|c|c|c|c|c|c|c|c|c|}
\hline \multicolumn{9}{|c|}{ Caracterização Física } \\
\hline Areia & Silte & Argila & $M_{o}$ & $f_{m}$ & $\varepsilon$ & $\rho_{b}$ & $\rho_{p}$ & \multirow{2}{*}{$\begin{array}{c}\text { Classificação } \\
\text { textural }\end{array}$} \\
\hline & & $\%$ & & & $\bar{Z}$ & \multicolumn{2}{|c|}{$\mathrm{kg} \mathrm{m}^{-3}$} & \\
\hline 81,632 & 3,645 & 14,723 & 3,382 & 59,118 & 37,500 & 1600 & 2560 & Franco Arenoso \\
\hline \multicolumn{9}{|c|}{ Ensaio de Infiltração } \\
\hline$\theta_{0}$ & $\theta_{f}$ & \multicolumn{3}{|c|}{$\begin{array}{l}\text { Tempo Total de } \\
\text { Infiltração }\end{array}$} & \multicolumn{3}{|c|}{ Infiltração Cumulativa } & $\begin{array}{c}\text { Taxa de Infiltração } \\
\text { Média }\end{array}$ \\
\hline \multicolumn{2}{|c|}{$\mathrm{mm}^{3} \mathrm{~mm}^{-3}$} & \multicolumn{3}{|c|}{$\mathrm{s}$} & \multicolumn{3}{|c|}{$\mathrm{mm}$} & $\mathrm{mm} \mathrm{s}^{-1}$ \\
\hline 0,016 & 0,374 & \multicolumn{3}{|c|}{2552} & \multicolumn{3}{|c|}{91,200} & 0,110 \\
\hline
\end{tabular}

Tabela 1. Parâmetros obtidos a partir da caracterização física e do ensaio de infiltração do solo.

Fonte: Autores.

Os parâmetros descritos na Tabela 1 subsidiaram o emprego do BEST, possibilitando a determinação dos parâmetros de forma $(n, m$ e $\eta)$ e de normalização $\left(\theta_{s}, k_{s}\right.$ e $\left.h_{g}\right)$ do solo, os quais estão presentes na Tabela 2.

Tabela 2. Parâmetros de forma ( $n, m$ e $\eta)$ e de normalização $\left(\theta_{s}, k_{s}\right.$ e $\left.h_{g}\right)$ do solo. 
Research, Society and Development, v. 10, n. 5, e54910515398, 2021

(CC BY 4.0) | ISSN 2525-3409 | DOI: http://dx.doi.org/10.33448/rsd-v10i5.15398

\begin{tabular}{cccccc}
\hline$n$ & $m$ & $\eta$ & $\theta_{s}$ & $k_{s}$ & $h_{g}$ \\
\hline & - & & $\mathrm{mm}^{3} \mathrm{~mm}^{-3}$ & $\mathrm{~mm} \mathrm{~s}^{-1}$ & $\mathrm{~mm}$ \\
\hline 2,263 & 0,116 & 10,608 & 0,375 & 0,040 & $-16,277$ \\
\hline
\end{tabular}

Fonte: Autores.

Utilizando-se dos resultados descritos anteriormente, apresenta-se na Figura 3 as curvas de condutividade hidráulica $K(\theta)(\mathrm{A})$ e de retenção de água no solo $\theta(h)(\mathrm{B})$.

Figura 3. Curvas de condutividade hidráulica $K(\theta)(\mathrm{A})$ e retenção de água no solo $\theta(h)(\mathrm{B})$.
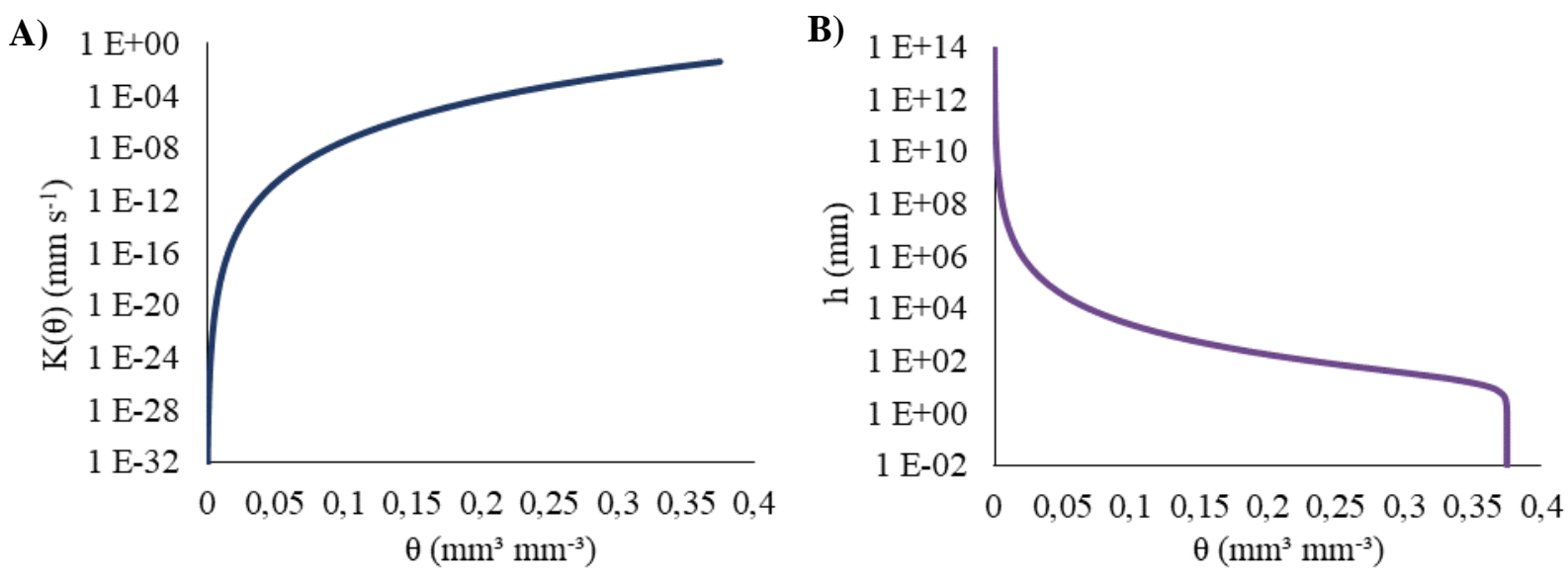

Fonte: Autores.

Mediante o emprego das metodologias propostas por De Vries (1963), foram obtidas as propriedades térmicas do solo em relação a umidade volumétrica. $\lambda$ variou entre $0,88 \mathrm{~W} \mathrm{~m}^{-1} \mathrm{k}^{-1}$, para o solo seco, e $2,23 \mathrm{~W} \mathrm{~m}^{-1} \mathrm{k}^{-1}$, para o solo em condição saturada, já $\mu$ variou de $1,3510^{6} \mathrm{~J} \mathrm{~m}^{-3} \mathrm{~K}^{-1}$, solo seco, a $2,7910^{6} \mathrm{~J} \mathrm{~m}^{-3} \mathrm{~K}^{-1}$, solo saturado, e $\alpha$ teve sua variação entre $0,6510^{-6}$ $\mathrm{m}^{2} \mathrm{~s}^{-1}$ e $0,8510^{-6} \mathrm{~m}^{2} \mathrm{~s}^{-1}$. Na Figura 4 estão presentes as curvas de $\lambda$ (A), $\mu$ (B) e $\alpha$ (C) em função de $\theta$. 
Figura 4. Variação da condutividade térmica aparente $(\lambda)(\mathrm{A})$, da capacidade térmica volumétrica $(\mu)(\mathrm{B})$ e da difusividade térmica aparente $(\alpha)(C)$ em função da umidade volumétrica do solo $(\theta)$, a partir do modelo de De Vries (1963)
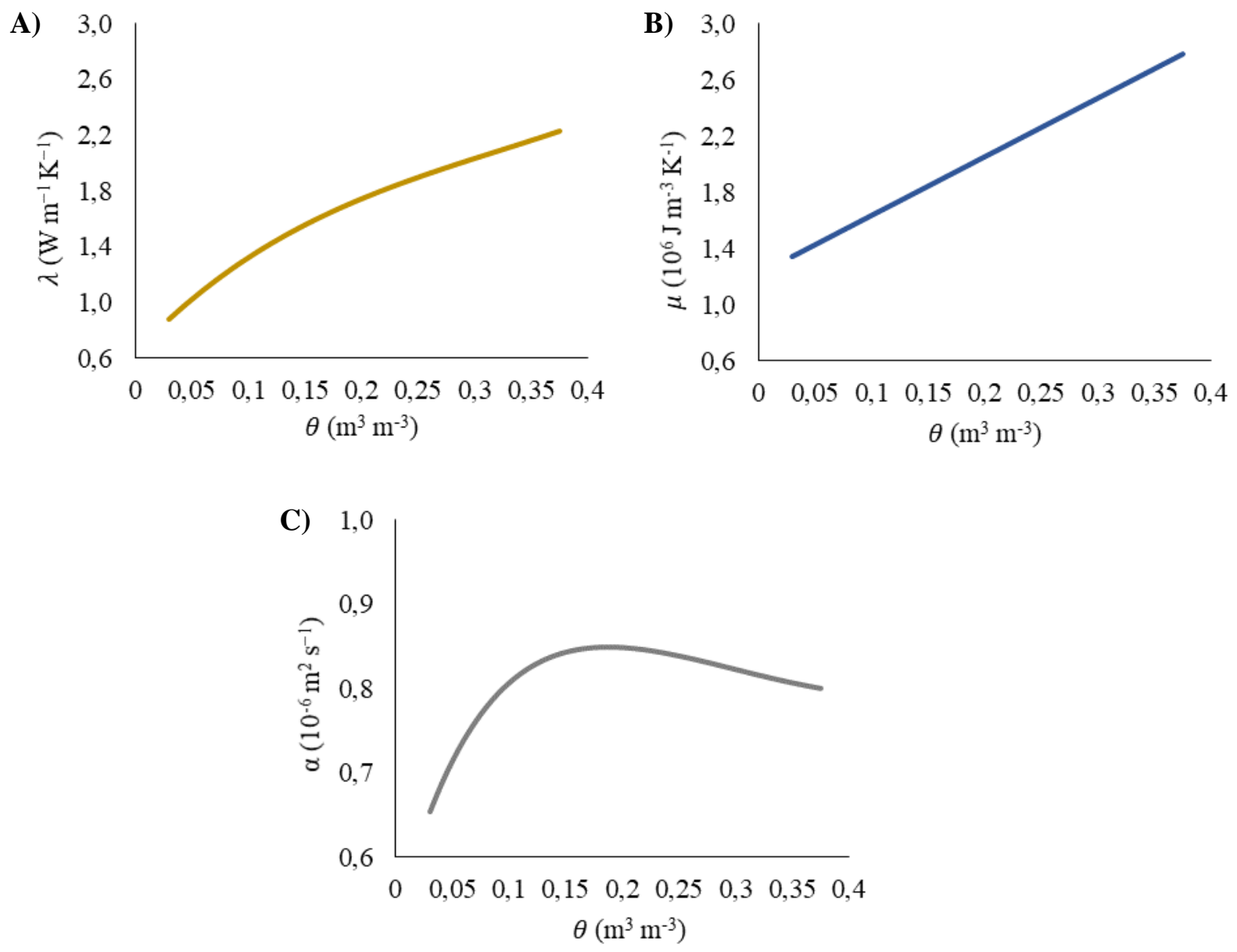

Fonte: Autores.

\section{Discussão}

Sales (2015) utilizou o BEST para caracterização hidrodinâmica de solos de Mata Atlântica presentes na bacia hidrográfica do rio Gramame, Paraíba. Comparando os valores dos parâmetros apresentados na Tabela 2 com os obtidos pela autora, observou-se que os valores médios obtidos para $n, 2,555$, e para $m, 0,214$, foram relativamente superiores, enquanto que para o parâmetro $\eta$ foi encontrado um valor médio de 6,951. Lassabatèrre et al. (2006), ao analisarem os parâmetros $n, m$ e $\eta$ para diferentes classes texturais, verificaram um crescimento tênue dos valores de $n$ e $m$ para solos mais grossos, sendo o inverso observado para $\eta$. Tendo em vista os elevados percentuais de areia encontrados por Sales (2015) em comparação com o presente trabalho, verificasse a concordância dos valores apresentados. Com relação aos parâmetros de normalização, a autora encontrou uma variação para $k_{s}$ entre 0,009 e $0,299 \mathrm{~mm} \mathrm{~s}^{-1}$ e para $h_{g}$ entre $-90,418$ e $-9,708 \mathrm{~mm}$.

Macêdo e Soares (2020) determinaram as propriedades hidrodinâmicas de um solo Pernambucano em condição urbanizada, obtendo valores médios ligeiramente inferiores para os parâmetros $m$ e $n(0,083$ e 2,181, respectivamente) e um valor médio relativamente superior para $\eta(13,559)$. Já para os parâmetros $k_{s}$ e $h_{g}$ os autores obtiveram, respectivamente, $0,171 \mathrm{~mm} \mathrm{~s}^{-1}$ e $-0,056 \mathrm{~mm}$. Os autores comentam que os elevados valores obtidos para $k_{s}$ e $h_{g}$ deu-se pelo fato de que em solos urbanos, quando ocorre precipitação, boa parte da água é escoada superficialmente, devido à presença de superfícies 
impermeáveis, ao passo que as áreas com cobertura vegetal apresentam capacidade de infiltração superior, estando essa maior capacidade de infiltração intrinsicamente relacionada com o tipo de uso do solo e a classe textural do mesmo.

Em termos de caracterização térmica, as mudanças no comportamento termodinâmico do solo mediante a variação de umidade podem ser atribuídas às diferenças na condução de calor nos próprios materiais do solo e à formação de pontes de capilaridade entre as partículas do mesmo. Conforme explicitado por Ewing e Horton (2007), para solos relativamente secos, a água é mantida firmemente nas superfícies das partículas do solo por forças adesivas e $\lambda$ permanece quase constante, uma vez que a pequena quantidade água contida nos poros contribui de forma insignificante para a condução de calor. À medida que $\theta$ aumenta, as partículas do solo são progressivamente revestidas com água e pontes de capilaridade são formadas entre as partículas sólidas. Consequentemente, um aumento de $\theta$ amplia a área de contato entre as partículas sólidas e a água, desloca o ar e melhora a condução de calor.

Em um estudo comparativo, Carvalho et al. (2013) analisaram o comportamento térmico de um solo sob floresta e pastagem no leste da Amazônia. Os autores utilizaram o método da amplitude para obtenção de $\alpha$ e observaram sua variação em uma camada de 0,05 a 0,20 m, tanto no período seco, quanto no chuvoso, e obtiveram uma média sazonal global de 0,87 $10^{-6} \mathrm{~m}^{2} \mathrm{~s}^{-1}$ na pastagem e $0,7610^{-6} \mathrm{~m}^{2} \mathrm{~s}^{-1}$ na floresta. Já para os valores de $\lambda$ os autores obtiveram uma variação de 0,67 a 0,82 $\mathrm{W} \mathrm{m} \mathrm{m}^{-1} \mathrm{~K}^{-1}$ para a pastagem e 1,51 a 3,31 $\mathrm{W} \mathrm{m}^{-1} \mathrm{~K}^{-1}$ para floresta. Observou-se que o solo sob floresta apresenta uma espessa camada de serrapilheira que forma uma proteção para o solo, dificultando sua perda de água, não deixando o mesmo perder a mesma quantidade de água do solo sob pastagem. No solo coberto pressupõe-se que haja maior teor de umidade e essa altera o calor específico e a condutividade térmica do solo.

Zhao e Si (2019) utilizaram os modelos propostos por De vries (1963) para realizar a caracterização térmicas de solos diversos, encontrando valores médios para $\mu$, $\lambda$ e $\alpha$, em solos arenosos, variando de 1,15 a $3,2510^{6} \mathrm{~J} \mathrm{~m}^{-3} \mathrm{~K}^{-1}, 0,60$ a $2,80 \mathrm{~W} \mathrm{~m}$ ${ }^{1} \mathrm{~K}^{-1}$ e 0,54 a $1,2010^{-6} \mathrm{~m}^{2} \mathrm{~s}^{-1}$, respectivamente, estando os valores obtidos no presente trabalho dentro destes limites. Os autores observaram que as propriedades térmicas sofrem um significativo aumento conforme crescem os teores de areia, $\theta \mathrm{e}$ $\rho_{b}$, porém observou-se que $\lambda$ é mais afetado pela composição do solo, enquanto $\mu$ parece mais controlado por $\theta$. Em contraste, $\alpha$ apresenta, inicialmente, uma tendência crescente, atingindo um valor de pico e, em seguida, exibindo uma tendência decrescente com um novo aumento de $\theta$. Na maioria dos solos, o pico de $\alpha$ é obtido com valores superiores a $20 \%$ de umidade por volume do solo, o que corrobora com os resultados apresentados na Figura 4 (C), tendo $\alpha$ atingido o pico quando $\theta=0,185$ $\mathrm{m}^{3} \mathrm{~m}^{-3}$.

\section{Conclusão}

Diante dos resultados desta pesquisa, é possível concluir que a metodologia utilizada se mostrou satisfatória para realização da caracterização hidrodinâmica e térmica do solo estudado, localizado no bairro de Dois Irmãos, Recife, Pernambuco, Brasil.

Por meio do BEST, mediante ensaios de infiltração e granulometria realizados em laboratório, foram obtidos valores coerentes para os parâmetros de forma $(n, m$ e $\eta)$ e de normalização $\left(\theta_{s}, k_{s}\right.$ e $\left.h_{g}\right)$ das curvas de retenção da água no solo e de condutividade hidráulica, estando estes valores dentro da faixa estabelecida na literatura.

As propriedades térmicas do solo foram obtidas a partir dos modelos propostos por De Vries (1963), o qual se mostrou bastante eficiente para obtenção destas propriedades, ficando evidente que a água no solo desempenha um papel fundamental na dinâmica da propagação do calor. 


\section{Referências}

NBR 7181. Associação brasileira de normas técnicas. (2016). Solo: análise granulométrica. 13 p.

NBR 13600. Associação brasileira de normas técnicas. (1996). Solo: Determinação do teor de matéria orgânica por queima a $440{ }^{\circ}$ C. 2 p.

Brooks, R. H. \& Corey, A. T. (1964). Hydraulic properties of porous media. Hydrology Paper, Colorado.

Burdine, N. T. (1953). Relative permeability calculations from pore size distribution data. Journal of Petroleum Technology, 5(3), 71-78. http://dx.doi.org/10.2118/225-g

Carneiro, R. G., Moura, M. A., Silva, V. D. P., Silva Junior, R. S., Andrade, A. \& Santos, A. B. D. (2014). Variabilidade da temperatura do solo em função da liteira em fragmento remanescente de mata atlântica. Revista Brasileira de Engenharia Agrícola e Ambiental, 18(1), 99-108. http://dx.doi.org/10.1590/S141543662014000100013

Carvalho, S. P., Souza, J. R. S. \& Makino, M. (2013). Observações e estimativas de propriedades térmicas do solo sob floresta e pastagem no leste da Amazônia. Revista Brasileira de Meteorologia, 28(3), 331-340. https://doi.org/10.1590/S0102-77862013000300009

Castellini, M., Di Prima, S., Moret-Fernández, D. \& Lassabatere, L. (2021). Rapid and accurate measurement methods for determining soil hydraulic properties: A review. Journal of Hydrology and Hydromechanics, 69, 1-19. https://doi.org/0.2478/johh-2021-0002

De Vries, D. A. (1963). Thermal Properties of Soil. In van Wijk, W. R (Ed.), Physics of Plant Environment (pp. 210-233) North Holland.

Ewing, R. \& Horton, R. (2007). Thermal conductivity of a cubic lattice of spheres with capillary bridges. Journal of Physics: Applied Physics, 40(16), p. 49594965. https://doi.org/10.1088/0022-3727/40/16/031

Freitas, J. P. O. D., Dias, H. C. T., Barroso, T. H. A. \& Poyares, L. D. B. Q. (2013). Distribuição da água de chuva em Mata Atlântica. Revista Ambiente \& Água, 8(2), p. 100-108. https://doi.org/10.4136/ambi-agua.1141

Haverkamp, R., Ross, P. J., Smettem, K. R. J. \& Parlange, J. Y. (1994). Three-dimensional analysis of infiltration from the disc infiltrometer. 2. Physically based infiltration equation. Water Resources Research, 30, 2931-2935. https://doi.org/10.1029/94WR01788

Hillel, D. (1998). Environmental soil physics. Academic Press, 771p.

Kelleners, T. J., Koonce, J., Shillito, R., Dijkema, J., Berli, M., Young, M. H., Frank, J. M. \& Massman, W. J. (2016). Numerical modeling of coupled water flow and heat transport in soil and snow. Soil Science Society of America Journal, 80(2), p. 247-263. https://doi.org/10.2136/sssaj2015.07.0279

Lassabatère, L., Angulo-Jaramillo, R., Soria, J. M., Cuenca, R., Braud, I. \& Haverkamp, R. (2006). Beerkan estimation of soil transfer parameters through infiltration experiments - BEST. Soil Science Society of America Journal, 70, 521-532. https://doi.org/10.2136/sssaj2005.0026

Lima, M. S. D., Freire, F. J., Marangon, L. C., Almeida, B. G. D., Ribeiro, E. P. \& Santos, R. L. D. (2018). Solos florestais em fragmento de floresta urbana na Mata de Dois Irmãos, Recife, Pernambuco, Brasil. Ciência Florestal, 28(2), 542-553. https://doi.org/10.5902/1980509832037

Macêdo, G. \& Soares, W. (2020). Utilização de métodos de campo e laboratoriais para estimação de propriedades hidrodinâmicas do solo. Águas Subterrâneas, 34(2), 166-176. https://doi.org/10.14295/ras.v34i2.29809

Sales, E. G. (2015). Impacto das Culturas da Cana-de-Açúcar e do Abacaxi nas Propriedades Hidrodinâmicas do Solo da Bacia do Rio Gramame-PB. Águas Subterrâneas, 27(3).

Santana, R. O., Delgado, R. C. \& Schiavetti, A. (2020). The past, present and future of vegetation in the Central Atlantic Forest Corridor, Brazil. Remote Sensing Applications: Society and Environment, 20, e100357. https://doi.org/10.1016/j.foreco.2019.117591

Teixeira, P. C., Donagemma, G. K., Fontana, A. \& Teixeira, W. G. (2017). Manual de métodos de análise de solo. (3a ed.), Embrapa Solos, 573 p.

Tian, Z., Lu, Y., Horton, R. \& Ren, T. (2016). A simplified de Vries-based model to estimate thermal conductivity of unfrozen and frozen soil. European Journal of Soil Science, 67(5), 564-572. https://doi.org/10.1111/ejss.12366

Van Genuchten, M. T. (1980). A closed-form equation for predicting the conductivity of unsaturated soils. Soil Science Society of America Journal, 44(5), 892-897. https://doi.org/10.2136/sssaj1980.03615995004400050002x

Zhao, Y. \& Si, B. (2019). Thermal properties of sandy and peat soils under unfrozen and frozen conditions. Soil and Tillage Research, 189, 64-72. https://doi.org/10.1016/j.still.2018.12.026

Zhao, Y., Si, B., Zhang, Z., Li, M., He, H. \& Hill, R. L. (2019). A new termal conductivity model for sandy and peat soils. Agricultural and Forest Meteorology, 274, 95-105. https://doi.org/10.1016/j.agrformet.2019.04.004 